\title{
Transatlantica
}

Revue d'études américaines. American Studies Journal

\section{Joan Mitchell, Peintures}

Musée des Impressionnismes, Giverny, 23 août-31 octobre 2009.

\section{Kamila Benayada}

\section{CpenEdition}

Journals

Édition électronique

URL : https://journals.openedition.org/transatlantica/4555

DOI : 10.4000/transatlantica.4555

ISSN : 1765-2766

Éditeur

Association française d'Etudes Américaines (AFEA)

Référence électronique

Kamila Benayada, « Joan Mitchell, Peintures », Transatlantica [En ligne], 2 | 2009, mis en ligne le 03 janvier 2010, consulté le 31 janvier 2023. URL : http://journals.openedition.org/transatlantica/4555 ; DOI : https://doi.org/10.4000/transatlantica.4555

Ce document a été généré automatiquement le 31 janvier 2023.

\section{cc) (†) $९$}

Creative Commons - Attribution - Pas d'Utilisation Commerciale - Pas de Modification 4.0 International - CC BY-NC-ND 4.0

https://creativecommons.org/licenses/by-nc-nd/4.0/ 


\title{
Joan Mitchell, Peintures
}

Musée des Impressionnismes, Giverny, 23 août-31 octobre 2009.

\author{
Kamila Benayada
}

1 Le nouveau Musée des Impressionnismes à Giverny présente des œuvres de Joan Mitchell (1926-92) pour sa deuxième exposition, qui confirme son ambition de montrer à la fois des œuvres impressionnistes, et leur impact sur l'art du XXe siècle.

2 Les toiles de Joan Mitchell, dont la plupart furent peintes en France, viennent de l'exposition itinérante "La peinture des deux mondes", organisée à Emden en Allemagne, puis à Reggio Emilia en Italie. Elles datent des années 1950 jusqu'à la fin de la vie de l'artiste, et sont accompagnées d'un rappel biographique assez complet, ainsi que de citations de l'artiste et de critiques d'art, et d'un film d'Angeliki Haas de la série «American Artists in Europe » (1976).

3 Les œuvres, réparties en quatre salles, suivent un itinéraire chronologique, partant de toiles du début des années cinquante, souvent sans titre, où l'on voit le travail du peintre dans la spontanéité, le geste ample, la fusion des pâtes épaisses et des coulées de peinture diluée, qui lui ont garanti la reconnaissance du critique Clement Greenberg et son inclusion dans le rang des peintres expressionnistes abstraits ${ }^{1}$. Pourtant, le travail du peintre est encore centré, la couleur et le geste s'arrêtent avant les bords, le blanc du fond encadre la couleur, se fond même avec la toile nue, l'œil est rassuré. La seconde salle, consacrée aux œuvres datant de la fin des années cinquante jusqu'aux années soixante-dix, propose des toiles dans la continuation de celles de la première salle. Mais avec les années soixante apparaissent les diptyques, et des toiles dont le titre les place directement dans la tradition du paysage. Les œuvres gagnent en monumentalité en même temps qu'elles deviennent plus lisibles, que figure la flore du jardin normand de l'artiste, et que la couleur atteint les bords, semblant vouloir les dépasser. Les polyptiques, pourtant, font obstacle à la lecture directe, en fragmentant le figuré. Le Tilleul (1978) est une œuvre où l'artiste peint un arbre tout à fait reconnaissable, et le coupe en son milieu. L'œuvre peut atteindre des dimensions plus grandes grâce à l'emploi de deux toiles, mais en même temps, l'artiste joue sur l'effet de miroir, la reproduction, la continuité, la linéarité de la lecture, la juxtaposition d'entités à la fois indépendantes et associées. Dans la troisième salle, deux œuvres des 
années soixante-dix atteignent des dimensions énormes, prennent tout le mur, font exploser la figuration. Les Bleuets (1973) n'en sont plus, ils sont taches de couleur. Le fond est surface, travaillée sur différentes couches, dans la transparence ou l'opacité.

4 Le musée a fait le choix d'établir un lien entre ces œuvres et celles de Monet, notamment en incluant la toile Nymphéas avec Rameau de Saule dans cette troisième salle, en suggérant une similarité des pratiques des deux peintres, fondées sur l'observation de la nature, l'intérêt pour la lumière et la couleur, la surface picturale monumentale et sans point de fuite. Mais Mitchell elle-même refusait ce parallèle, préférant ancrer son oeuvre et sa pratique dans la mémoire plutôt que dans la vision elle-même. Mitchell peint le souvenir des paysages, et le souvenir des sentiments qu'ils avaient produits en elle. Elle ne peint pas la chose vue, mais l'empreinte cérébrale et émotionnelle de la chose. L'œil est déclassé, la mémoire l'emporte. En cela, Mitchell est véritablement une artiste du XXe siècle. Si l'exposition la rapproche des expressionnistes abstraits, et surtout de Pollock et de De Kooning, cet intérêt pour le travail du souvenir la rapproche de Stuart Davis, qui tentait de reproduire sur la toile non pas ce qu'il avait observé, mais l'émotion gardée de l'observation, la trace de la nature. La monumentalité des œuvres des deux dernières salles contribue à cette victoire de l'abstrait, du cérébral. Les titres, Un Jardin pour Audrey (1979), Tournesols (1990-91), Champs (1990), sont une référence directe à la nature, mais la taille des œuvres nie la figuration. Là encore, les polyptiques «to be seen in one piece » disait l'artiste, font de ces paysages reconstitués des images faites de fragments, comme la mémoire opérant en assemblant des fragments de souvenirs pour n'en faire qu'une image, souvent déformée.

Mais Mitchell garde les pieds sur terre, ses œuvres y sont ancrées, et cette verticalité, accentuée par les coulées de peinture trop diluée, marquant l'irrésistible force de la gravité, les distancie des œuvres purement abstraites. Mitchell peignait à la verticale, pas à l'horizontale comme Pollock. Et par là, le sens de la lecture est imposé, jusque dans les œuvres les moins « lisibles ». Les traces de la mémoire en sont un déplacement, le paysage remplaçant la nature. Rosalind Kraus, citée dans l'exposition, met le doigt sur la distance qu'il semble y avoir entre l'ambition du peintre et les œuvres ellesmêmes :

By taking a vocabulary of shape used in the context of a pictorial displacement of space, and importing it for reuse in an illusionistic game of replacement (a full size painting of nature replaces a piece of nature), Mitchell has made a set of monumental works that seem both grandiose and dry. ${ }^{2}$

Mitchell, employant une syntaxe abstraite, reste dans l'illusion. La monumentalité de ses œuvres est certes un jeu sur l'espace, mais dans la mesure où elle garde cet appui dans la figuration, l'espace qu'elle crée sur la toile serait une transposition de l'espace naturel, où la toile remplace la nature, sans la changer. Pour Kraus, cela rend la peinture de Mitchell «sèche", presque redondante. Le grandiose vient non pas du contenu pictural, mais simplement de la taille.

7 Le jugement est sévère. Il fait de Mitchell un peintre de paysages, et non pas un peintre abstrait, dans un Amérique qui voulait trouver dans l'abstraction sa particularité (et peut-être sa justification) esthétique. Cela explique peut-être la réticence de l'artiste à se voir affiliée à Monet. Par ailleurs, Kraus néglige peut-être l'importance du jeu qu'opère Mitchell entre surface et profondeur. En effet, si le peintre tend vers la non illusion, la non reproduction du réel tel que l'œil le perçoit, jusque dans le choix de 
titres neutres (sans titre), négation de toute illusion représentative, elle garde de la profondeur tout en travaillant à la surface de la toile. Dès les années cinquante, dans les premières toiles que l'exposition nous montre, les œuvres sont à la fois plates et elles emmènent l'œil vers un fond illusoire. La Chatière (1960) en témoigne : le centre où s'accumulent les couleurs attire l'œil, l'entraîne vers un au-delà de la toile, et ce nœud de couleur agit comme la chatière, en ouvrant vers un ailleurs imaginaire. En gardant la couleur au centre de la toile, Mitchell suggère un cadre pour le "sujet ", même si ce «sujet » est le travail esthétique du peintre abstrait. Paradoxalement, l'artiste échappe à cet encadrement de son propos et de son geste de peintre dans les toiles où la nature revient en évidence. Mais là, la re-présentation de la nature est annulée par la forme du polyptique. La toile est alors espace multiple, multiplié; il n'est pas tant déplacé, comme le suggère Kraus, que recréé. Un simple déplacement suppose qu'il n'y ait aucune altération. Mais l'espace de la toile est autre : en ne s'arrêtant pas à la surface, comme le voudrait l'abstraction, Mitchell ouvre vers l'imaginaire. Partant du souvenir, de la trace de la nature dans sa mémoire, elle ouvre vers un ailleurs.

En cela, cette exposition remplit l'ambition que se donne le nouveau musée de Giverny : montrer les liens qui mènent de l'impressionnisme à l'art du XXe siècle, où encore les racines de l'art abstrait dans l'art de la fin du XIXe siècle. Mitchell, artiste expressionniste abstrait, reconnue par Clement Greenberg, a une peinture empreinte de souvenirs de la nature, qui dépasse la vision optique, et mène le spectateur au-delà de ce que l'œil perçoit.

\section{NOTES}

1. Mitchell a exposé dans la même galerie que Joseph Cornell ou Rauschenberg, et dans l'exposition organisée par le critique et historien de l'art Meyer Schapiro consacrée à la seconde génération d'expressionnistes abstraits en 1957.

2. In « Painting becomes Cyclorama », Artforum vol.12 n¹0, June 1974, p.52.

\section{INDEX}

Thèmes : Trans'Arts

\section{AUTEUR}

\section{KAMILA BENAYADA}

Université d'Orléans 